\title{
Valor da Taxa de Eritroblastos no Sangue da Veia Umbilical de Recém- Nascidos como Marcador Hematológico da Hipóxia Perinatal
}

\author{
Importance of Erythroblast Count in the Newborn's Umbilical Vein as a Hematological \\ Marker For Perinatal Hypoxia \\ Ivo Behle, Rogério Carapeto Fernandes, Celze Clarde Mousquer Calderon Peres \\ Débora Wildholzer, Sérgio Moreira Espinosa, Lucia Pellanda Zimmer, Cristiane Kopaceck Zilz
}

\begin{abstract}
RESUMO
Objetivo: examinar se a taxa de eritroblastos, no sangue da veia umbilical de recém-nascidos, tem relação com a hipoxia perinatal, analisada pelos parâmetros que expressam o equilíbrio ácido-básico (EAB) do sangue funicular.

Métodos: de recém-nascidos vivos com pelo menos 37 semanas de gestação, assistidos no Hospital de Alvorada-RS, foram coletadas amostras de sangue da veia umbilical antes da instalação da respiração. Parte do sangue foi coletado em frasco contendo EDTA, determinando-se as séries vermelha e branca. No sangue coletado em seringa com heparina, foram determinados valores do $\mathrm{pH}, \mathrm{pO}_{2}, \mathrm{pCO}_{2}$ e calculado o EAB. Em lâmina corada pelo corante panótico, procedeu-se à contagem manual do número de eritroblastos. A taxa de eritroblastos foi calculada em relação ao número de leucócitos.

Resultados: dos 158 casos que compõem o estudo, em 55 as condições perinatais permitiram considerá-los como isentos de acometimento de processo hipóxico. A média da taxa de eritroblastos foi 3,9\%, com o desvio-padrão de 2,8\%. Os valores minimo e máximo foram 0\% e 10\%, respectivamente. Dentre os 158 casos, a taxa dos eritroblastos foi 5,7\%, com desviopadrão de 5,3\%. Os valores mínimo e máximo foram $0 \%$ e $28 \%$, respectivamente. A aplicação do teste de Pearson a taxa dos eritroblastos e valores dos parâmetros do EAB mostrou correlação significativa para o $\mathrm{pH}$ e $\mathrm{pCO}_{2}$. A elaboração da curva $\mathrm{ROC}$ revelou que $5 \%$ de eritroblastos e $\mathrm{pH}$ de 7,25 representam pontos de corte que contrabalançam a sensibilidade e especificidade (54\% e 56\%), respectivamente. Dos 23 conceptos com taxa de eritroblastos maior que 10\%, 7 (30,4\%) estavam acidóticos, 11 (48,7\%) eram grandes para a idade gestacional, 3 (13\%) eram pequenos para a idade gestacional, $7(30,4 \%)$ tinham anemia e em $3(13 \%)$ não foram constatadas anormalidades.

Conclusões: em recém-nascidos de gestações e partos sem complicações, a taxa de eritroblastos ao nascimento foi menor do que 10\%. Quando a taxa de eritroblastos foi maior do que 10\% houve correlação principalmente com acidemia, distúrbios do crescimento intra-uterino $e$ anemia fetal.
\end{abstract}

PALAVRAS-CHAVE: Eritroblastos. Hipoxia perinatal, Gravidez normal. Vitalidade fetal.

Hospital Alvorada - Fundação Universitária de Cardiologia do Rio Grande do Sul

Instituto de Cardiologia do Rio Grande do Sul

Correspondência:

Ivo Behle

Unidade de Pesquisa do IC/FUC -

Av. Princesa Isabel, 395 - Santana

90620-001 - Porto Alegre - RS

Fone/Fax.: (51) 230-3600 Ramal 3777

email: behle@terra.com.br

\section{Introdução}

A paralisia cerebral ocorre em um ou dois casos para cada 1.000 recém-nascidos a termo ${ }^{1,2}$. Como conseqüência da asfixia fetal intraparto, entretanto, ela é evento raro, com incidência referida de um ou dois casos para cada 10.000 nas- 
cidos a termo ${ }^{2}$. Devido às repercussões emocionais e financeiras que determina na familia e na sociedade, médicos e pesquisadores buscam definir meios para sua prevenção, bem como procuram determinar as suas causas ${ }^{3}$.

Para cada criança que nasce com asfixia, duas perguntas se impõem - quando ocorreu o episódio hipóxico e qual a sua causa. As respostas estão relacionadas com os conhecimentos que temos sobre as repercussões que a asfixia determina sobre o tecido nervoso fetal. Durante muito tempo se acreditou que a grande maioria das paralisias cerebrais decorria de lesões ligadas ao parto ${ }^{4}$. Esta concepção alimentou, principalmente em países desenvolvidos, dispendiosos processos judiciais, sendo responsáveis pelo afastamento de muitos obstetras da atividade clínica. Hoje se sabe que mesmo em situações catastróficas, tais como na rotura uterina ou no prolapso de cordão, que podem determinar intensa e prolongada hipóxia, nem sempre ocorre lesão do tecido nervoso central. Por outro lado, situações que passam às vezes desapercebidas, principalmente durante o período pré-natal, são capazes de lesar irremediavelmente o cérebro do concepto $^{1,2,5}$.

Os resultados dos estudos realizados em animais são limitados pelo fato de não existir modelo com comportamento semelhante ao do feto humano em face da hipóxia. No feto humano, quando o nivel de saturação do oxigênio alcança $10 \%$, geralmente ocorre processo da centralização sangüínea ${ }^{6}$. Sabe-se que ela pode permanecer assim por muito tempo sem que haja comprometimento neuronal. Isto depende da ocorrência ou não de hipotensão bem como das reservas de que o feto dispõe, ambos de dificil caracterização.

Estes novos conhecimentos têm impulsionado pesquisadores a buscar marcadores capazes de identificar conceptos submetidos a processo asfixico, bem como sinalizar o momento de sua ocorrência, com finalidade de aperfeiçoar a assistência perinatal. A identificação de um marcador capaz de apontar conceptos que foram submetidos a insultos antes do parto auxiliaria, também, na tomada de decisões nos processos judiciais.

A presença de células sangüineas vermelhas nucleadas (CSVN), também conhecidas por eritroblastos e normoblastos, no sangue funicular e na circulação periférica do neonato parece ser um dos indicadores mais valiosos da ocorrência de evento hipóxico. Há trinta anos, $\mathrm{Fox}^{7}$ propôs que o número de eritroblastos devia ser encarado como expressão da intensidade da deprivação do oxigênio. Soothill et al. ${ }^{8}$, por meio da cordocentese, estabeleceram correlação entre o número de CSVN por 100 leucócitos e a gravidade da hipoxemia, em fetos com crescimento intrauterino retardado. Phelan e $\mathrm{Ahn}^{2}$, Phelan et al. ${ }^{9}$ e Korst e Phelan ${ }^{1}$ concluíram que a presença de normoblastos no sangue do neonato deve ser encarada como importante marcador hematológico da asfixia perinatal, auxiliando no reconhecimento do momento da ocorrência do processo lesivo. Naeye e Localio ${ }^{10}$ demonstraram que além dos eritroblastos, também o número de linfócitos é capaz de identificar a ocorrência do processo asfixico.

Dentre os fatores que reconhecidamente se relacionam com taxas elevadas de eritroblastos estão a prematuridade ${ }^{11}$, o diabete melito descompensado ${ }^{12}$, a anemia fetal ${ }^{8}$ e o crescimento fetal deficiente ${ }^{8}$. Recentemente foi postulado que a taxa de normoblastos pode ser maior em crianças de mães com condições socioeconômicas deficientes ${ }^{13}$.

Objetivamos nesta pesquisa determinar a taxa de eritroblastos no sangue da veia umbilical de recém-nascidos considerados isentos de terem sido submetidos a processo hipóxico perinatal, cujos partos foram assistidos num hospital regional. Também, numa coorte de recémnascidos não selecionados, objetivamos determinar se a taxa de eritroblastos no sangue da veia umbilical se correlaciona com a vitalidade ao nascimento, traduzida pelos parâmetros que expressam o estado ácido-básico do sangue funicular.

\section{Métodos}

Dos recém-nascidos de parturientes que acessaram espontaneamente o Hospital de Alvorada, na região metropolitana de Porto Alegre, Rio Grande do Sul, entre os meses de julho e dezembro de 1999, foram coletadas amostras de sangue funicular imediatamente após a expulsão fetal e antes da instituição da respiração. Foram dispensados da coleta os natimortos, os prematuros, quando a premência da expulsão fetal impediu o preparo do material ou quando a mãe não concordou em participar da pesquisa. O sangue, coletado por punção da veia umbilical, livre de bolhas, foi acondicionado num frasco com EDTA e também numa seringa de insulina, cujas paredes haviam sido lavadas com heparina, e encaminhado ao laboratório. Foram determinados os valores do $\mathrm{pH}, \mathrm{pO}_{2}, \mathrm{pCO}_{2}$ e calculado o estado ácido-básico em aparelho de gasimetria Ciba-Corning248 com eletrodo seletivo. Considerou-se presença de acidemia quando o 
$\mathrm{pH}$ foi menor do que 7,25 e o déficit de bases igual ou maior do que $-6 \mathrm{mEq} / \mathrm{L}^{14}$. Do frasco com EDTA foram avaliadas as séries vermelha e branca em analisador hematológico Spirit (Biochem Immunosistems Inc-USA). Uma fração deste sangue foi utilizada para confeccionar esfregaço corado pelo corante panótico rápido. Os normoblastos foram quantificados visualmente, expressos em porcentagem sobre o total dos leucócitos. Foram realizadas três aferições por profissionais diferentes e o resultado expressa a média obtida entre eles. Assim, leucócitos totais representa o número de leucócitos da amostra, por $\mathrm{mm}^{3}$, calculado descontando o número absoluto de eritroblastos na amostra do número global de células contadas pelo aparelho hematológico; o valor de eritroblastos absolutos representa o número de eritroblastos por $\mathrm{mm}^{3}$, calculado subtraindo a percentagem de eritroblastos do número global de células contadas pelo aparelho hematológico; a porcentagem de eritroblastos é determinada em lâmina, numa distensão da amostra, corada com corantes hematológicos, sobre uma população global de células, compreendendo leucócitos e eritroblastos.

Os antecedentes familiares, pessoais e tocoginecológicos, bem como as características do parto e do período neonatal imediato, foram coletados da história clínica perinatal simplificada (HCPS), do Centro Latino-Americano de Perinatologia (CLAP) da OMS, empregada rotineiramente na instituição. Os dados aí digitados foram analisados pela aplicação de Programa SIP6, que permitiu cotejar o peso de nascimento com a idade gestacional, distribuindo-o segundo percentis.

Do total de 158 casos arrolados durante o período descrito, 55 preencheram os seguintes critérios: a gestação transcorreu sem anormalidades e, portanto, foi rotulada de baixo risco; o trabalho de parto teve início espontâneo, transcorreu sem uso de medicamentos e teve duração menor do que 12 horas; o período de rotura das membranas não ultrapassou 18 horas; o parto foi normal; não houve sinais clínicos (presença de mecônio) nem cardiotocográficos (alterações da freqüência cardíaca fetal basal) de estado fetal não-tranquilizador ${ }^{15}$; o indice de Apgar foi igual ou maior do que 7 no primeiro e quinto minutos de vida; o $\mathrm{pH}$ da veia umbilical foi igual ou maior do que 7,25 e o déficit de bases não foi maior do que $-6 \mathrm{mEq} / \mathrm{L}$; o hematócrito do sangue da veia umbilical foi igual ou maior do que 40\%; a avaliação do recém-nascido, pelo método de Capurro, caracterizou idade entre 37 e 41 semanas; o exame neurológico ao nascimento foi normal; o peso ao nascimento situouse entre os percentis 50 e 75 para idades entre 37 e 41 semanas respectivamente, de uma curva elaborada a partir da população de 2.875 crianças nascidas no Hospital de Alvorada, no período de outubro de 1998 a outubro 1999, pela aplicação do Programa SIP6; não houve intercorrências no período neonatal e a alta da criança coincidiu com a da mãe.

Os recém-nascidos destes casos foram considerados como isentos de terem sido submetidos a processo hipóxico perinatal.

Todos os dados foram inseridos em programa elaborado com o pacote epidemiológico EpiInfo (Versão 6.04, OMS) e posteriormente analisados por meio de programa estatístico SPSS (versão 8.0). A descrição de todas as variáveis foi realizada mediante de médias e desvio-padrão, para variáveis numéricas, e proporções para variáveis categóricas. Os valores dos eritroblastos foram correlacionados às variáveis referentes ao equilibrio ácido-básico pela correlação de Pearson. Foi considerado estatisticamente significativo nível de $95 \%(\mathrm{p}=<0.05)$.

O presente estudo foi aprovado pelo Comitê de Ética em Pesquisa da Fundação Universitária de Cardiologia (UP 1492/99).

\section{Resultados}

Na Tabela 1 estão representados os valores minimos, máximos, as médias e os desviospadrão da paridade, idade gestacional, peso ao nascimento e índices de Apgar do primeiro ao quinto minutos, dos 158 casos estudados. Estas caracteristicas definem a amostra como representativa de uma população assistida numa unidade de atenção primária.

Tabela 1 - Dados obstétricos e perinatais dos 158 casos estudados.

\begin{tabular}{lccccc}
\hline Parâmetro & Paridade & Idade gestacional & Peso ao nascimento & Apgar ao $\mathbf{1}^{\mathbf{0}}$ minuto & Apgar ao $\mathbf{5}^{\mathbf{0}}$ minuto \\
\hline Mínimo & 0,00 & 37,00 & 1620,00 & 1,00 & 4,00 \\
Máximo & 10,00 & 41,00 & 4450,00 & 10,00 & 10,00 \\
Média & 1,56 & 39,24 & 3255,82 & 8,50 & 9,26 \\
Desvio-padrão & 1,83 & 1,21 & 479,12 & 1,17 & 0,89 \\
\hline
\end{tabular}


Na Tabela 2 estão apontados os valores mínimos, máximos, médias e desvios-padrão do $\mathrm{pH}$, $\mathrm{pO}_{2}, \mathrm{pCO}_{2}, \mathrm{BE}$ (excesso ou déficit de bases) e taxa de eritroblastos dos 158 casos estudados. Também aqui os valores obtidos representam aqueles esperados para amostra com as características já assinaladas.

Tabela 2 - Valores dos eritroblastos e dos parâmetros do equilíbrio ácido-básico dos 158 casos estudados

\begin{tabular}{lcrrrc}
\hline Parâmetro & $\mathbf{p H}$ & $\mathrm{pO}_{2}$ & $\mathrm{pCO}_{2}$ & \multicolumn{1}{c}{$\mathrm{BE}$} & Eritroblastos \\
\hline Mínimo & 6,71 & 7,50 & 23,30 & $-20,40$ & 0,00 \\
Máximo & 7,44 & 42,10 & 152,70 & 3,20 & 28,00 \\
Média & 7,29 & 23,08 & 47,94 & $-3,94$ & 5,70 \\
Desvio-padrão & 0,09 & 6,86 & 12,44 & 3,32 & 5,34 \\
\hline
\end{tabular}

A Figura 1 representa a distribuição das freqüências da taxa de eritroblastos nos 158 casos estudados.

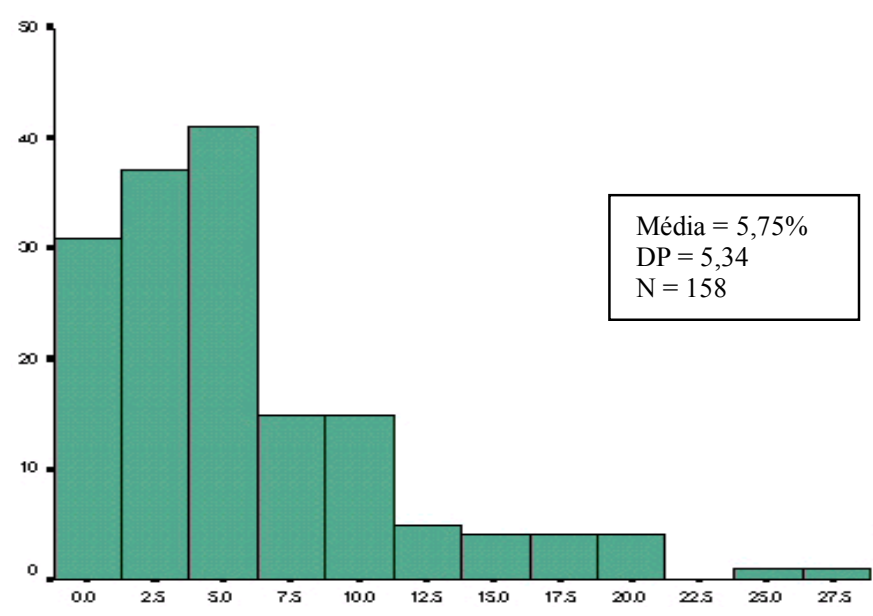

Figura 1 - Distribuição das freqüências dos eritroblastos nos 158 casos estudados.

A Figura 2 expressa os valores estatísticos dos eritroblastos na população total bem como nos 55 casos considerados como representantes da normalidade, no que se refere à vitalidade dos conceptos. Dos 158 casos que compõem o estudo, em 55 as condições perinatais permitiram considerá-los como isentos de acometimento de processo hipóxico. A média da taxa de eritroblastos foi $3,9 \%$, com o desvio-padrão de $2,8 \%$. Os valores mínimo e máximo foram $0 \%$ e $10 \%$, resectivamente. Dentre os 158 casos, a taxa dos eritroblastos foi $5,7 \%$, com desvio padrão de $5,3 \%$.
Os valores mínimo e máximo foram $0 \%$ e $28 \%$, respectivamente. A dispersão em torno da média, na população total, é bem maior do que na população considerada isenta de processo hipóxico.

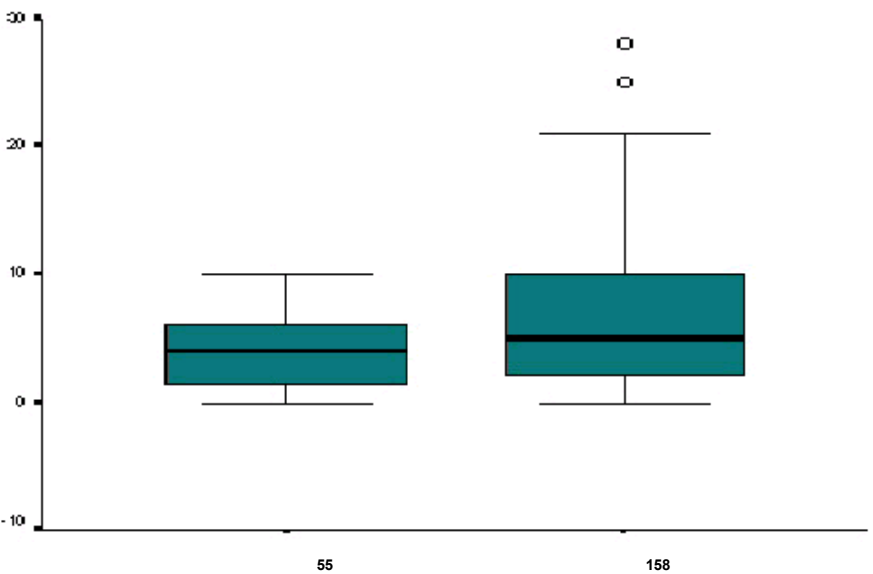

Figura 2 - Valores estatísticos obtidos na população de 158 casos bem como nos 55 representantes da normalidade perinatal.

A Figura 3 expressa a curva ROC (receiver operator characteristic curve), que traduz o contrabalanço entre sensibilidade e especificidade da taxa de eritroblastos para ponto de corte do $\mathrm{pH}$ em 7,25 e BE em $-6 \mathrm{mEq} / \mathrm{L}$.

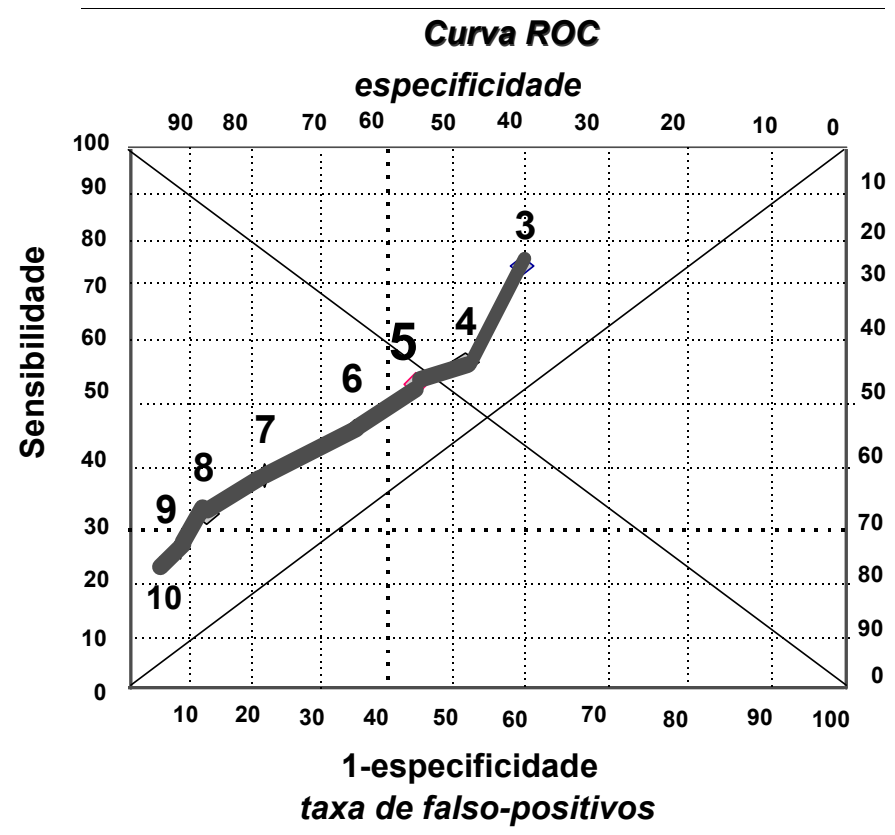

Figura 3 - Contrabalanço entre sensibilidade e especificidade na detecção da acidemia fetal. 
A aplicação do teste de Pearson a taxa dos eritroblastos e valores dos parâmetros do EAB mos- trou correlação significativa para o $\mathrm{pH}$ e $\mathrm{pCO}_{2}(\mathrm{Ta}-$ bela 3$)$.

Tabela 3 - Resultados obtidos pela aplicação do teste de correlação de Pearson nos 158 casos.

\begin{tabular}{lcccccrr}
\hline Parâmetro & $\mathbf{p H}$ & $\mathrm{pO}_{2}$ & $\mathbf{P C O}_{2}$ & $\mathrm{~EB}$ & Peso & Apgar 1 & Apgar 5 \\
\hline Pearson & $-0,281^{*}$ & $-0,164^{*}$ & $0,227^{*}$ & $-0,150$ & 0,095 & $-0,206^{*}$ & $-0,150$ \\
Significância & 0,000 & 0,040 & 0,004 & $-0,061$ & 0,236 & 0,010 & 0,059 \\
\hline
\end{tabular}

* significante em nível de 0,01

\section{Discussão}

Soothill et al. ${ }^{8}$ verificaram que em fetos normais a taxa de eritroblastos raramente ultrapassa $10 \%$ dos leucócitos totais. Pesquisando sangue fetal colhido por cordocentese, documentaram que há correlação entre a taxa dos eritroblastos e a gravidade da hipoxemia quando da ocorrência de crescimento intra-uterino deficiente.

Postula-se que o feto responde à deprivação do oxigênio não só derivando o fluxo de sangue de órgãos menos vitais para o cérebro, coração e adrenais, mas também estimulando a produção da eritropoetina, que ativa a eritropoiese. Isto faz com que exista aumento de células sangüíneas vermelhas jovens, ainda nucleadas.

O comportamento dos eritroblastos, nos 55 recém-nascidos considerados como isentos de terem sido submetidos a processo hipóxico perinatal, está de acordo com os resultados encontrados por Korst e Phelan ${ }^{1}$, Phelan e Ahn ${ }^{2}$ e Naeye e Localio ${ }^{10}$. Em apenas dois casos a taxa de CSVN atingiu 10\%. O primeiro corresponde a recém-nascido cujo peso ficou próximo ao limite superior para a idade gestacional. Não podemos deixar de considerar a eventualidade de este concepto ter sido submetido a incremento na oferta de glicose durante a gravidez, decorrente da intolerência materna aos hidratos de carbono, não identificada pela propedêutica habitual. Recentemente, demonstrou-se que a taxa de eritroblastos encontra-se aumentada em recém-nascidos de mães diabéticas metabolicamente não-controladas ${ }^{16}$. O segundo se refere ao concepto com menor peso ao nascimento. Aqui, ao contrário, não se pode excluir a possibilidade de ele ter sido submetido a crescimento intra-uterino deficiente, já que o peso verificado está muito próximo do limite inferior do $50^{\circ}$ percentil da curva empregada. Os resultados obtidos pela análise destes casos permite-nos concluir que em recém-nascidos a termo, com vitalidade normal, de gestações sem intercorrências, cujos partos transcorreram sem complicações, a taxa de eritroblastos no sangue da veia umbilical, colhido antes da instalação da efetiva respiração, não ultrapassa $10 \%$. Esta conclusão é também referendada por Phelan et al. ${ }^{9}$.

A aplicação do teste de Pearson nos 158 casos estudados permitiu verificar que a taxa de eritroblastos correlacionou-se com os valores do $\mathrm{pH}$ e do $\mathrm{pCO}_{2}$. A elaboração da curva ROC revelou que $5 \%$ representa a taxa de eritroblastos que expressa o contrabalanço entre a especificidade e a sensibilidade para o ponto de corte do $\mathrm{pH}$ em 7,25 e BE em $-6 \mathrm{mEq} / \mathrm{L}$. Se considerarmos apenas os valores encontrados para a especificidade (56\%) e sensibilidade (54\%), o teste não pode ser considerado como portador de elevado poder discriminatório. Entretanto, interessa relevar algumas considerações. Pesquisas realizadas com finalidade de decifrar o comportamento dos eritroblastos, diante de injúrias que determinaram paralisias cerebrais, revelaram que é de aproximadamente duas horas o tempo necessário para que o processo hipóxico-acidêmico determine a passagem das CSVN para a circulação fetal. O monitoramento neonatal seriado dos eritroblastos revelou que este processo tende a persisitir até 36 horas após o nascimento e que também as maiores taxas se relacionam com processos asfixicos graves $(\mathrm{pH}$ abaixo de 7,00 e $\mathrm{BE}$ maior do que $-20 \mathrm{mEq} / \mathrm{L})^{1,2,15}$. Nestes casos estão presentes também sinais secundários de asfixia, representados por indices de Apgar inferiores a 3 , necessidade de assistência ventilatória imediata e sinais precoces de encefalopatia hipóxica, como convulsões, coma e falência de múltiplos órgãos ${ }^{15}$. Salvo situações excepcionais, tais como rotura uterina, descolamento prematuro da placenta ou prolapso do cordão umbilical, a ocorrência de processos asfixicos com esta gravidade não é determinada pelo processo do parto em si. Por outro lado, existem outras evidências que apontam situações capazes de determinar hipoxemia e acidose, com menor repercussão sobre o $\mathrm{pH}$ e déficit de bases, como capazes de determinar lesão neuronal ${ }^{1}$. Trata-se de conceptos que chegam ao parto com reservas baixas, decorrentes de processos hipóxicos que 
incidiram no período pré-natal. Como os parâmetros do equilíbrio ácido-básico no sangue funicular se correlacionam apropriadamente com o estado da vitalidade ao nascimento, a existência de um marcador que seja capaz de apontar conceptos que estiveram lidando com processos de compensação no período que antecede o parto, como por exemplo a centralização do volume sangüíneo circulante, encontra respaldo especulativo. Quando analisamos os 23 casos que apresentaram taxa de eritroblastos maior do que $10 \%$, que representa o valor da média acrescido de um desvio padrão, verificamos que houve acidemia em 7 (30,4\%), em $11(47,8 \%)$ os conceptos eram grandes para a idade gestacional, em $3(13,0 \%)$ os conceptos eram pequenos para a idade gestacional, em $7(30,4 \%)$ houve anemia fetal e em $3(13,0 \%)$ não se evidenciou nenhuma anormalidade. Abstraindo estes últimos três, em $20(12,65 \%)$ havia pelo menos um fator de risco capaz de ter determinado aumento na taxa dos eritroblastos do sangue funicular.

A escolha da veia umbilical para a coleta de sangue funicular deveu-se a três fatores. Em primeiro lugar, a veia é de mais fácil identificação e manuseio. Em segundo lugar, os valores dos eritroblastos podem ser cotejados com aqueles descritos por Soothill et al. ${ }^{8}$, coletados por cordocentese, e que portanto traduzem a situação fetal anteparto. Em terceiro lugar, as diferenças entre os valores dos parâmetros do sangue venoso e arterial não comprometem o emprego clínico dos resultados ${ }^{14}$.

Por se tratar de metodologia simples, de fácil execução e não dispendiosa, os resultados obtidos induzem-nos a concordar com aqueles que a preconizam $^{1,2,4,17}$, associada com a determinação dos parâmetros do equilíbrio ácido-básico como forma de aperfeiçoar a expressão das condições ao nascimento.

\section{SUMMARY}

Purpose: nucleated red blood cell counts are increased in several hypoxic conditions. The authors aimed to establish if there is a correlation between erythroblast counts in the umbilical vein of newborns and the presence of perinatal hypoxia detected by acid-base balance parameters.

Methods: blood samples were obtained from the umbilical vein of pregnant subjects with at least 37 weeks of gestation attended at the Hospital de Alvorada-RS, just before the newborns' first breathing movement. Part of the blood was placed in an EDTA-containing vial and white and red blood cells were analyzed. The remaining amount of blood was aspirated into insulin type syringe previously washed with heparin and $\mathrm{pH}, \mathrm{pO}_{2}, \mathrm{pCO}_{2}$ and acid-base excess/deficit were analyzed. Slides were also prepared with the panoptic stain for visual identification and count of the erythroblast number. The erythroblast/leukocyte ratio was calculated.

Results: of the 158 cases included in the study, 55 were considered free of perinatal hypoxia. In this population, the average erythroblast rate was $3.9 \%$ with a standard deviation of $2.8 \%$. The minimum and maximum values were $0 \%$ and $10 \%$, respectively. When considering all the cases, the average was $5.7 \%$, with a standard deviation of $5.3 \%$. The minimum and maximum values were $0 \%$ and $28 \%$, respectively. Application of Pearson's test for the analysis of the erythroblast rate and acid-base parameters showed a significant correlation for $\mathrm{pH}$ and $\mathrm{pCO}$. The construction of a Receiver Operation Curve showed that for an erythroblast rate of 5\%, a 7.25-pH cutoff yields a sensitivity of $54 \%$ and a specificity of $56 \%$. Out of the 23 newborns whose normoblast rate was greater than $10 \%$, there was acidemia in $7(30.4 \%)$, $11(48.7 \%)$ were large for gestational age, 3 (13\%) were small for gestational age, 7 (30.4\%) were anemic, and in 3 (13\%) there were no abnormalities.

Conclusions: in newborns from uncomplicated pregnancies and deliveries, the erythroblast rate was less than $10 \%$. When it was greater than $10 \%$, a correlation was found mainly with large or small for gestational age, fetal anemia and acidemia.

KEY-WORDS: Normal pregnancy. Fetal well-being. Perinatal hypoxia. Nucleated red blood cells.

\section{Agradecimentos}

Aos médicos plantonistas: Ana Lucia Brum Castro, Ana Lucia Semmelmann, Barbara Canabarro Frantz, Elcio Starck Machado, Ellen Regina Kuse, Fernando Mariano Obst, Fernando Pecis, Glenise de Andrade, Helio Antonio Rossi Castro, Janine Cavagnolli, Lisiane Neila Saginn, Luciana Slongo Coiro e Rodrigo Celicato.

\section{Referências}

1. Korst LM, Phelan JP. Hematologic markers to date fetal neurologic injury. In: Maulik D, editor. Asphyxia and Fetal Brain Damage. 1st ed. New York: Wiley-Liss; 1999. p.159-70.

2. Phelan JP, Ahn MO. Perinatal observations in fortyeight neurologically impaired term infants. Am J Obstet Gynecol 1994; 171:424-31. 
3. Nelson KB, Dambrosia JM, Ting TY, Grether JK. Uncertain value of electronic fetal monitoring in predicting cerebral palsy. N Engl J Med 1996; 334:613-8.

4. Dear P, Newell S. Establishing probable cause in cerebral palsy. How much certainly is enough? BMJ 2000; 320:1075-6.

5. Naeye RL. How to time when hypoxic-ischemic fetal brain damage took place. In: Maulik D, editor. Asphyxia and Fetal Brain Damage. 1st ed. New York: Wiley-Liss; 1999. p.153-8.

6. Myers RE. Two patterns of perinatal brain damage and their conditions of occurrence. Am J Obstet Gynecol 1972; 112:246-76.

7. Fox H. The incidence and significance of nucleated erythrocytes in the foetal vessels of the mature human placenta. J Obstet Gynaecol Br Commonw 1967; 74:40-3.

8. Soothill PW, Nicolaides KH, Campbell S. Prenatal asphyxia, hyperlacticaemia and erythroblastosis in growth retarded fetuses. Br Med J (Clin Res Ed) $1987 ; 294: 1051-3$.

9. Phelan JP, Ahn MO, Korst LM, Martin GI. Nucleated red blood cells: a marker for fetal asphyxia? Am J Obstet Gynecol 1995; 173:1380-4.

10. Naeye RL, Localio AR. Determining the time before birth when ischemia and hypoxemia initiated cerebral palsy. Obstet Gynecol 1995; 86:713-9.
11.Anderson GW. Studies on the nucleated red blood cell count in the chorionic capillaries and the cord blood of various ages of pregnancy. Am J Obstet Gynecol 1941; 42:1-14.

12.Green DW, Mimouni F. Nucleated erythrocytes in healthy infants and in infants of diabetic mothers. J Pediatr 1990; 116:129-31.

13.Ellis M, Costello AML. Antepartum risk factors for newborn encephalopathy. BMJ 1999; 318:1414.

14.D'Souza SW, Black P, Cadman J, Richards B. Umbilical venous blood $\mathrm{pH}$ : a useful aid in the diagnosis of asphyxia at birth. Arch Dis Child 1983; 58:15-9.

15. MacLennan A. A template for defining a causal relation between acute intrapartum events and cerebral palsy: international consensus statement. BMJ 1999; 319:1054-9.

16.Yeruchimovich M, Mimouni FB, Green DW, Dollberg S. Nucleated red blood cells in healthy infants of women with gestational diabetes. Obstet Gynecol 2000; 95:84-6.

17.Buonocore G, Perrone S, Gioia D, et al. Nucleated red blood cell count at birth as an index of perinatal brain damage. Am J Obstet Gynecol 1999; 181:1500-5.

\title{
VIII Congresso de Ginecologia e Obstetrícia da Região Sudeste
}

Período: 27 a 30 de junho de 2001

\author{
Local:Vitória-ES
}

\section{Organização:SOGOES}

\section{Tel.: (27) 325-1765 / (27) 227-4468}

\section{e-mail: sogoes@ig.com.br}

